\title{
Similar progression of diabetic retinopathy with insulin glargine and neutral protamine Hagedorn (NPH) insulin in patients with type 2 diabetes: a long-term, randomised, open-label study. Reply to Chantelau E [letter] and Currie CJ [letter]
}

\author{
J. Rosenstock • V. Fonseca • J. B. McGill • M. Riddle • \\ J.-P. Hallé • I. Hramiak • P. Johnston • M. Davis
}

Received: 29 July 2009 / Accepted: 30 July 2009/Published online: 25 August 2009

(C) The Author(s) 2009. This article is published with open access at Springerlink.com

Keywords Insulin glargine - Neutral protamine Hagedorn ·

Retinopathy · Type 2 diabetes

$\begin{array}{ll}\text { Abbreviations } \\ \text { DR } & \text { Diabetic retinopathy } \\ \text { ETDRS } & \text { Early Treatment Diabetic Retinopathy Study } \\ \text { NPH } & \text { Neutral protamine Hagedorn } \\ \text { UKPDS } & \text { UK Prospective Diabetes Study }\end{array}$

To the Editor: We thank Dr E. Chantelau and Dr C. Currie for their interest [1, 2] in our findings [3], which we consider important because they are from a long-term randomised study that avoids the many limitations of

J. Rosenstock ( $\bowtie)$

Dallas Diabetes and Endocrine Center at Medical City,

7777 Forest Lane C-685,

Dallas, TX 75230, USA

e-mail: juliorosenstock@dallasdiabetes.com

J. Rosenstock

University of Texas Southwestern Medical School,

Dallas, TX, USA

V. Fonseca

Tulane University Medical Center,

New Orleans, LA, USA

\section{J. B. McGill}

Washington University School of Medicine,

St Louis, MO, USA

\section{Riddle}

Oregon Health and Science University,

Portland, OR, USA retrospective database registry studies. They mainly commented on the power of the study and the possibility of performing post hoc sub-analyses. We would like to address their assertions, which we believe to be incorrect or misleading.

We strongly disagree with Dr Chantelau's suggestion that the study was underpowered to detect changes in the rate of progression of diabetic retinopathy (DR) [1]. The sample size and power for the study were calculated based on these assumptions: a 20\% 5 year background event rate; a non-inferiority margin of $10 \%$ (i.e. $50 \%$ of the background rate of $20 \%$ ); approximately $60 \%$ of the randomised participants evaluable; and the two treatments being equivalent. Statistical power considerations are only rele-

J.-P. Hallé

Université de Montréal,

Montréal, QC, Canada

I. Hramiak

University of Western Ontario,

London, ON, Canada

P. Johnston

sanofi-aventis,

Bridgewater, NJ, USA

M. Davis

University of Wisconsin School of Medicine and Public Health, Madison, WI, USA 
vant during the study planning stage, to arrive at the sample size. On completion, the non-inferiority hypothesis will either be accepted or rejected, depending on the actual outcome observed, and the pre-study power assumption is no longer relevant. Nevertheless, had a 5 year background event rate of $15 \%$ been projected (our observed rates were 14.2\% and $15.7 \%$ in the insulin glargine [A21Gly,B31Arg,B32Arg human insulin] and neutral protamine Hagedorn [NPH] insulin groups, respectively), with a non-inferiority margin of $7.5 \%$ (i.e. $50 \%$ of the background $15 \%$ rate) and a sample size that is the same as that of the per protocol population, the study would have had $81 \%$ power to demonstrate equivalence. Hence, the study was not underpowered.

Dr Chantelau also questioned the low frequency of DR at study entry $(15.6 \%$ and $12.1 \%$ of patients reporting a medical history of DR in the insulin glargine and the NPH insulin groups, respectively; Table 1 of [3]), and objected to pooling patients with and without DR at baseline in assessing progression. Regarding the baseline prevalence of DR, Table 1 of our paper [3] also shows the frequency of DR based on grading of the baseline fundus photographs. By using this more sensitive measurement, a substantial proportion of patients were shown to have DR $(\sim 61 \%$ in both groups). Pooling patients with no DR and those with nonproliferative DR is appropriate when analysing, as we did, the prevalence of worsening by at least three steps on the Early Treatment Diabetic Retinopathy Study (ETDRS) retinopathy severity scale. To illustrate this, in the DCCT, this outcome was observed at 6 years in the primary prevention cohort (no DR) in $35 \%$ and $11 \%$ of the conventional and intensive treatment groups, respectively. The corresponding rates in the secondary prevention cohort (mild to moderate nonproliferative DR) were similar-31\% and $11 \%$, respectively [4]. In a subset of 1919 UK Prospective Diabetes Study (UKPDS) patients with appropriate photographs, the 6 year prevalence of worsening by three steps or more was $17.4 \%$ in 1216 patients without DR at baseline and $18.5 \%$ in 701 patients with non-proliferative DR [5].

Dr Currie [2] questions the selection of the patient population and the exclusion of established proliferative DR in the study, comments on the protective effects of metformin against cancer, proposes multiple arbitrary post hoc subgroup analyses, and notes that insulin glargine and NPH insulin resulted in similar glycaemic control. We believe that the population selected is indeed the population that should be studied, as it represents the majority of patients with type 2 diabetes treated with insulin who are seen in clinical practice. The entrance criteria allowed inclusion of a wide range of patients previously treated with human basal insulin, with or without regular insulin and oral agents; the resulting population closely matches that seen in clinical practice. The entry criteria did specify a particular range of DR, to assure a population in which assessment of the primary outcome would be most effective. The observed event rate for progression of three or more steps in ETDRS score in this study was $\sim 15 \%$ at 5 years [3], and was similar to the rates in the UKPDS and DCCT [4, 5], as described above. Therefore, the suggestion that '... these patients could be those patients least likely to develop more severe visual loss ...' is incorrect.

Inclusion of patients with advanced forms of DR to assess progression, as Dr Currie suggested, is highly unreliable. In this study, no statistically significant difference in the development of proliferative DR was observed between the two groups, despite a greater frequency of the highest category of non-proliferative DR - in which progression of proliferative DR is most likely - at entry in the insulin glargine group.

The comparison of our randomised trial with a retrospective analysis on metformin and cancer is inappropriate. The percentage of patients using metformin was similar in both groups in this study $(41 \%$ at baseline in the glargine group vs $42 \%$ with $\mathrm{NPH}$, and $18 \%$ started after randomisation in the glargine group vs $16 \%$ with NPH) [3]; therefore, an imbalance of the effects of metformin could not have contributed to the findings. Furthermore, the publication authored by Currie et al. [6] and cited in his letter [2] actually demonstrated no increase in the frequency of cancer with insulin glargine compared with other insulins. There is no basis for Dr Currie's speculative statement that 'Akin to the safety issue with respect to cancer, it could be that insulin glargine does not trigger the [retinal] pathology, but, rather, promotes or accelerates the pathological process.' Moreover, the question of the potential protective effects of metformin against cancer is difficult to evaluate in epidemiological analyses such as the ones Dr Currie cites, because of the tendency for metformin to be prescribed for younger and healthier patients. Regarding cancer risk with different insulins, our study is particularly relevant as it is the only randomised study to compare insulin glargine with human insulin, and because it shows no trend towards a greater risk of neoplasia with insulin glargine [7]. Recently published expert statements and objective criticisms have highlighted the possibilities for bias inherent in epidemiological, non-randomised comparisons of treatments [8-10].

The multiple subgroup analyses suggested by Dr Currie seem ill-advised for a randomised trial, in that (1) the diminished sample sizes of the subsets, with consequently much wider confidence intervals for statistical testing, weaken the conclusions that can be drawn; and (2) multiple post hoc analyses increase the chance of 'uncovering' findings that are statistically significant but spurious. Such post hoc sub-analyses can generate hypotheses, but neither provide final answers nor put to bed any lingering concerns.

Dr Currie notes that the slightly higher level of $\mathrm{HbA}_{1 \mathrm{c}}$ observed with insulin glargine compared with NPH insulin 
was not emphasised in the paper. The study design clearly states the retinal primary objective and emphasises that testing differences in glycaemic control between the two insulins was not one of the study objectives. The intention was for glycaemic control to be as similar as possible in the two groups, despite insulin glargine being given once daily and NPH insulin twice daily, to avoid confounding the interpretation of the retinal outcomes. The requirement that NPH insulin be given twice daily vs once daily for insulin glargine actually provides a bias in favour of NPH insulin in titration of dosage. The small difference $(0.2 \%)$ at study end in favour of NPH insulin is, therefore, not surprising. In any case, the upper bound of the two-sided $95 \%$ confidence interval for $\mathrm{HbA}_{1 \mathrm{c}}$ treatment difference was $0.35 \%$, which is within the non-inferiority margin of $0.4 \%$ conventionally accepted as a basis for clinical equivalence in efficacy of $\mathrm{HbA}_{1 \mathrm{c}}$ between two treatments. This difference may be important mainly in strengthening the finding that retinopathy was not adversely affected by use of insulin glargine vs NPH insulin, despite slightly higher mean glucose. Finally, concerning hypoglycaemia, this study confirms findings from many other studies, which have consistently found a lower incidence of hypoglycaemia with insulin glargine treatment than with NPH insulin with otherwise similar regimens and at similar levels of glycaemic control [11-18].

In summary, we believe that the assertions made in these two letters are speculative and misguided. Our findings and conclusions are based on the largest and longest randomised trial comparing two types of insulin. The study design was appropriate to address concerns about the effects that insulin glargine might have on the progression of diabetic retinopathy, and the conclusions are well supported. We are confident that the reassuring findings and conclusions are correct as reported.

Acknowledgements Technical editorial support was provided through the Global Publications Group of sanofi-aventis.

Duality of interest J. Rosenstock has received grants for research from and/or has been a consultant to Amylin, Boehringer-Ingelheim, BristolMyers Squibb, Centocor, Eli Lilly, Emisphere, GlaxoSmithKline, Johnson \& Johnson, MannKind, Merck, Novartis, Novo Nordisk, Pfizer, Roche, Sankyo, sanofi-aventis and Takeda. V. Fonseca has received research support (to Tulane University) with grants from GlaxoSmith Kline, Novartis, Novo Nordisk, Takeda, Astra-Zeneca, Pfizer, sanofiaventis, Eli Lilly, Daiichi-Sankyo, Novartis, the National Institutes of Health (NIH) and the American Diabetes Association (ADA), and honoraria for consulting and lectures from GlaxoSmithKline, Novartis, Takeda, Pfizer, sanofi-aventis and Eli Lilly. J. McGill has received grant support (to Washington University) from sanofi-aventis, Pfizer, Eli Lilly, Novo Nordisk, Novartis, GlaxoSmithKline, Elixir, Tolerx, Biodel, MannKind, Takeda; served on advisory boards and speakers' bureaus for sanofi-aventis and Novo Nordisk, and on speakers' bureaus for Eli Lilly, Merck, Novartis, Daiichi-Sankyo, Forest and GlaxoSmithKline; and has been a consultant/advisor for Merck, Novo Nordisk, Elixir,
MannKind and Amgen. I. Hramiak has received research grant support from Pfizer, sanofi-aventis, Novo Nordisk and Eli Lilly, and has served on advisory boards for GlaxoSmithKline, Novo Nordisk, sanofi-aventis and Merck. J.-P. Hallé has received grants for research from and/or has been a consultant and/or on the speaker bureau of Bristol-Myers Squibb, ConjuChem, Bellus Health, Eli Lilly, GlaxoSmithKline, Merck Frosst, Novartis, Novo Nordisk, Pfizer, Roche, sanofi-aventis and Takeda. M. Riddle has received grants for research and/or honoraria for consulting or lectures from Amylin, Lilly, the Amylin-Lilly Alliance, Novo Nordisk, Pfizer, sanofi-aventis and Valeritas. P. Johnston is an employee of, and holds stock options from sanofi-aventis. M. Davis has received research support from sanofi-aventis.

Open Access This article is distributed under the terms of the Creative Commons Attribution Noncommercial License which permits any noncommercial use, distribution, and reproduction in any medium, provided the original author(s) and source are credited.

\section{References}

1. Chantelau E (2009) Diabetic retinopathy and insulin glargine. Diabetologia. doi:10.1007/s00125-009-1488-3

2. Currie CJ (2009) The longest ever randomised controlled trial of insulin glargine: study design and $\mathrm{HbA}_{1 \mathrm{c}}$ findings. Diabetologia. doi:10.1007/s00125-009-1477-6

3. Rosenstock J, Fonseca V, McGill JB et al (2009) Similar progression of diabetic retinopathy with insulin glargine and neutral protamine Hagedorn (NPH) insulin in patients with type 2 diabetes: a long-term, randomised, open-label study. Diabetologia 52:1778-1788

4. The Diabetes Control and Complications Trial Investigators (1995) The effect of intensive diabetes treatment on the progression of diabetic retinopathy in insulin-dependent diabetes mellitus. Arch Ophthalmol 113:36-51

5. Stratton IM, Kohner EM, Aldington SJ et al (2001) UKPDS 50: risk factors for incidence and progression of retinopathy in type II diabetes over 6 years from diagnosis. Diabetologia 44:156-163

6. Currie CJ, Poole CD, Gale EAM (2009) The influence of glucoselowering therapies on cancer risk in type 2 diabetes. Diabetologia 52:1766-1777

7. Rosenstock J, Fonseca V, McGill JB et al (2009) Similar risk of malignancy with insulin glargine and neutral protamine Hagedorn (NPH) insulin in patients with type 2 diabetes: findings from a 5 year randomised, open-label study. Diabetologia 52:1971-1973

8. Expert statement issued about Lantus following recent publications in Diabetologia. Available from http://en.sanofi-aventis.com/ binaries/20090715 lantus consensus en tcm28-25675.pdf, accessed 24 July $20 \overline{0} 9$

9. Garg SK, Hirsch IB, Skyler JS (2009) Insulin glargine and cancer-an unsubstantiated allegation. Diabetes Technol Ther. doi:10.1089/ dia.2009.1705

10. Pocock SJ, Smeeth L (2009) Insulin glargine and malignancy: an unwarranted alarm. Lancet 374:511-513

11. Yki-Järvinen H, Kauppinen-Mäkelin R, Tiikkainen M et al (2006) Insulin glargine or NPH combined with metformin in type 2 diabetes: the LANMET study. Diabetologia 49:442-451

12. Rosenstock J, Schwartz SL, Clark CM Jr, Park GD, Donley DW, Edwards MB (2001) Basal insulin therapy in type 2 diabetes: 28-week comparison of insulin glargine (HOE 901) and NPH insulin. Diabetes Care 24:631-636 
13. Pan CY, Sinnassamy P, Chung KD, Kim KW (2007) Insulin glargine vs NPH insulin therapy in Asian type 2 diabetes patients. Diabetes Res Clin Pract 76:111-118

14. Massi Benedetti M, Humburg E, Dressler A, Ziemen M (2003) A one-year, randomised, multicentre trial comparing insulin glargine with NPH insulin in combination with oral agents in patients with type 2 diabetes. Horm Metab Res 35:189-196

15. Yki-Jarvinen H, Dressler A, Ziemen M (2000) Less nocturnal hypoglycemia and better post-dinner glucose control with bedtime insulin glargine compared with bedtime NPH insulin during insulin combination therapy in type 2 diabetes. HOE 901/3002 Study Group. Diabetes Care 23:1130-1136
16. Riddle MC, Rosenstock J, Gerich J (2003) The treat-to-target trial: randomized addition of glargine or human NPH insulin to oral therapy of type 2 diabetic patients. Diabetes Care 26:3080 3086

17. Eliaschewitz FG, Calvo C, Valbuena H et al (2006) Therapy in type 2 diabetes: insulin glargine vs NPH insulin both in combination with glimepiride. Arch Med Res 37:495-501

18. Fritsche A, Schweitzer MA, Häring HU, 4001 Study Group (2003) Glimepiride combined with morning insulin glargine, bedtime neutral protamine hagedorn insulin, or bedtime insulin glargine in patients with type 2 diabetes. A randomized, controlled trial. Ann Intern Med 138:952-959 